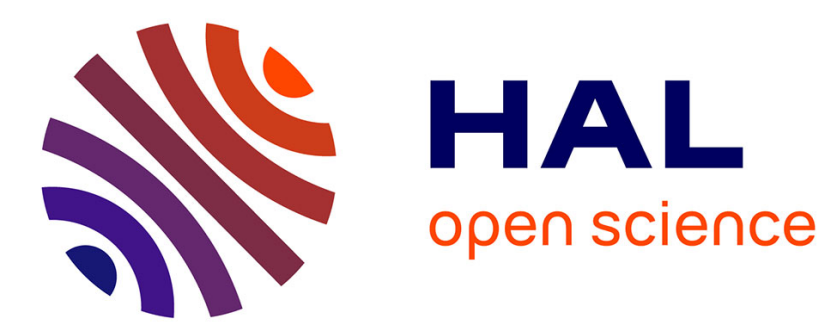

\title{
Second-order modeling for Rayleigh flat fading channel estimation with Kalman filter
}

\author{
Laurent Ros, Eric Pierre Simon
}

\section{To cite this version:}

Laurent Ros, Eric Pierre Simon. Second-order modeling for Rayleigh flat fading channel estimation with Kalman filter. DSP 2011 - 17th IEEE International Conference on Digital Signal Processing, Jul 2011, Corfou, Greece. 6 p. hal-00617586

\section{HAL Id: hal-00617586 \\ https://hal.science/hal-00617586}

Submitted on 29 Aug 2011

HAL is a multi-disciplinary open access archive for the deposit and dissemination of scientific research documents, whether they are published or not. The documents may come from teaching and research institutions in France or abroad, or from public or private research centers.
L'archive ouverte pluridisciplinaire HAL, est destinée au dépôt et à la diffusion de documents scientifiques de niveau recherche, publiés ou non, émanant des établissements d'enseignement et de recherche français ou étrangers, des laboratoires publics ou privés. 


\title{
SECOND-ORDER MODELING FOR RAYLEIGH FLAT FADING CHANNEL ESTIMATION WITH KALMAN FILTER
}

\author{
Laurent $\operatorname{ROS}^{1}$ and Eric-Pierre SIMON ${ }^{2}$ \\ 1-Gipsa-Lab, Image and Signal Department, BP 46, 38402 Saint Martin d'Heres, France \\ 2- IEMN lab, TELICE group, 59655 Villeneuve d'Ascq, France
}

\begin{abstract}
This paper deals with channel estimation over flat fading Rayleigh channel with Jakes' Doppler spectrum. Many estimation algorithms exploit the time-domain correlation of the channel by employing Kalman Filter based on an approximation of the time-varying channel. A common method used in the literature is based on a first-order auto-regressive (AR1) model for the channel approximation, combined with a Correlation Matching (CM) criterion to fix the value of the AR1-parameter. In this paper, we propose first to replace the AR1 model by a specific second-order (Or2) model (as the one used for the phase estimation in presence of frequency offset), which is more appropriate for slow fading variations. Secondly, we propose a criterion based on the Minimization of the Asymptotic Variance (MAV) of the Kalman estimator to fix the parameter of the Or2-model. Closed-form expressions of the optimum Or2-parameter and of the corresponding Mean Square Error (MSE) are derived for a given channel state (Doppler spread, SNR). MSE theoretical analysis and simulation results prove a significant improvement with the Or2-MAV approach compared to more conventional AR1-CM approach (or even AR1-MAV optimized approach) in terms of MSE, especially for slow fading variations.
\end{abstract}

Index Terms - Rayleigh Channel estimation, Jakes' Doppler spectrum, Flat fading, Kalman Filter, steady-state.

\section{INTRODUCTION}

The use of Kalman Filter (KF) for the channel estimation problem has received great attention in recent years in the wireless communication literature. It concerns a very large and various range of systems, as for example Multi-InputMulti-Output systems [3][4], or Orthogonal Frequency Division Multiplexing systems [5][6][7][10], or again GSM systems [9]. All the aforementioned works based their Kalman Filter on the autoregressive (AR) approximation of the widely accepted Rayleigh fading channel with Jakes' Doppler spectrum [15], as developed in [1]. Moreover, the tuning of the AR model parameters with respect to a given normalized Doppler frequency $\left(f_{d} T\right)$ is always based on a same criterion, called Correlation Matching (CM) criterion in this paper (see section
3.1.2). Note also that this AR-CM method to approach the correlated fading channels is also used today for the computer simulation of the "Rayleigh fading channel model with Jakes' Doppler spectrum", as for example in the actual version of Matlab (function "rayleighchan"). The authors of [1] insisted that low orders are appropriate for narrowband Doppler fading processes, and [2] showed that a first order assumption is enough to capture most of the channel tap dynamics. Finally, in the KF based channel tracking problem, the combination "AR1 model" (or Gauss-Markov assumption) and "CM criterion" is often retained [3][5][6][7][9]...

But for the case of slow fading scenario, the MSE results with the so-called AR1(CM)-Kalman estimator were somewhat disappointing (compared to Bayesian Cramer Rao Bound (BCRB), or to the MSE of lower complexity algorithms), as we pointed out recently in [10]-Fig.2 (see also [9]-Fig.3). It should be noted that the slow channel variations assumption $\left(f_{d} T \leq 10^{-2}\right)$ corresponds to the most common transmission scenario (e.g. [3][4][5][6]) where the channel variation within one symbol duration can be neglected (and then also the symbol distortion in a mono-carrier scenario, or the Inter-Carrier-Interference in a multi-carrier system [7]). Note also that some authors propose to modify slightly the $\mathrm{CM}$ constraint to better approximate the original process in some sense (for example [1] propose to add a positive $\varepsilon$ to the zero-th autocorrelation lag but without explicit analysis to fix the value of $\varepsilon$ ). The authors of $[11,12]$ explained analytically for the flat fading case the previous disappointing results and propose to compute the AR1-parameter under a Minimum Asymptotic Variance (MAV) criterion without imposing the CM constraint. The resulting MSE performance was then improved, but remains far from the BCRB.

In this paper, we propose a second-order model based KF designed for the flat fading Rayleigh channel estimation under the slow channel variations scenario. The 2nd-order model, called Or2-model in this paper, generalizes (for a complex parameter) the one used for the phase estimation scalar problem in presence of frequency offset [13][14]. This model is based on a Brownian evolution of the slope of the parameter to be tracked. The main motivation for this model is that for the slow fading Rayleigh channel $\left(f_{d} T \leq 10^{-2}\right.$ assumed), the channel Complex Gains (CGs) exhibit strong trend behavior, 
(slow fading case) $\operatorname{Re}\{\alpha(\mathrm{t})\}$, for $\boldsymbol{f}_{\boldsymbol{d}} \boldsymbol{T}=\boldsymbol{0 . 0 0 1}$



$\mathrm{t} / \mathrm{T}$ (fast fading case) $\operatorname{Re}\{\alpha(\mathrm{t})\}$, for $\boldsymbol{f}_{\boldsymbol{d}} \boldsymbol{T}=\mathbf{0 . 3}$



$\mathrm{t} / \mathrm{T}$
Fig. 1. Example (one realization) of the temporal evolution of a (Rayleigh-Jakes) Complex Gain (real part) over a window of 6 symbol durations, for a slow fading case (left, with $f_{d} T=$ $10^{-3}$ ), and a very fast fading case (right, with $f_{d} T=0.3$ )

i.e. they continue in some direction during several symbols, as shown in Fig.1-left. So it is satisfactory that the Or2-model takes into account a drift (or slope) into the CG variation contrary to the AR1-model, with a (slow) possible evolution of this slope. An important question that arises is about the choice (or tuning) of the Or2 model-parameter for a given channel state (Doppler frequency $f_{d} T$ of the Jakes' spectrum, Signal to Noise Ratio SNR). Considering the tracking mode where the KF can be regarded as a linear time-invariant filter, we give a MSE analysis that permits to express the asymptotic (or steady state) tracking error variance in terms of static and dynamic error variance. Then the Or2-parameter that permits to minimize the asymptotic global variance of the $\mathrm{KF}$ estimator (i.e. under MAV criterion) is derived. Simulation results and comparison to literature AR1-Kalman filters and to BCRB validate the proposed algorithm and the theoretical analysis.

This paper is organized as follows: section 2 describes the model and objectives, section 3 recalls Kalman Filters equations for AR1-KF and Or2-KF. Section 4 gives MSE analysis of the Or2-KF and the proposed optimization. Finally, the different results are discussed in Section 5.

\section{MODEL AND ESTIMATION OBJECTIVES}

We consider the estimation of a flat Rayleigh fading channel. The discrete-time observation is ${ }^{1}$ :

$$
y_{(n)}=\alpha_{(n)}+N_{(n)}
$$

where $n$ is the symbol time index, $N_{(n)}$ is a zero-mean additive white circular complex Gaussian noise with variance $\sigma_{N}^{2}$, and $\alpha_{(n)}$ is a zero-mean circular Gaussian channel CG

\footnotetext{
${ }^{1}$ Model (1) assumes that symbols are normalized and known (or decided), additionally to flat fading assumption. Although this model is admittedly simplistic, it can be applied to different (more involved) contexts, such as pilot-aided multicarrier systems in frequency-selective wireless channels.
}

with variance $\sigma_{\alpha}^{2}=1$. The normalized Doppler frequency of this channel is $f_{d} T$, where $T$ is the symbol period. A Jakes' Doppler spectrum is assumed for this channel:

$$
\Gamma_{\alpha}(f)= \begin{cases}\frac{\sigma_{\alpha}^{2}}{\pi f_{d} \sqrt{1-\left(\frac{f}{f_{d}}\right)^{2}}} & \text { if }|f|<f_{d} \\ 0 & \text { if }|f|>f_{d}\end{cases}
$$

The autocorrelation coefficient $R_{\alpha}[m]$ of the stationary CG $\alpha$ is then defined for lag $m$ by:

$$
R_{\alpha}[m]=\mathrm{E}\left\{\alpha_{(n)} \cdot \alpha_{(n-m)}{ }^{*}\right\}=\sigma_{\alpha}^{2} J_{0}\left(2 \pi f_{d} T \cdot m\right)
$$

where $J_{0}$ is the zeroth-order Bessel function of the first kind. Given the observation model (1) and the Doppler spectrum statistical constraint (2) for the dynamic evolution of the CG, we look for an on-line unbiased estimation $\hat{\alpha}_{(n)}$ of $\alpha_{(n)}$ based on KF. The variance $\sigma_{\varepsilon}^{2} \stackrel{\text { def }}{=} E\left\{\left|\varepsilon_{(n)}\right|^{2}\right\}$ of the estimation error $\varepsilon_{(n)} \stackrel{\text { def }}{=} \alpha_{(n)}-\hat{\alpha}_{(n)}$ will be investigated.

\section{KALMAN FILTERS}

\subsection{Review of the AR1-Kalman Filter}

\subsubsection{Dynamical model for approaching the $C G$ variation}

The time-varying CG $\alpha$ can be approached by a first order autoregressive (AR1) model $\alpha^{A R 1}$ :

$$
\alpha_{(n)}^{A R 1}=\gamma \cdot \alpha_{(n-1)}^{A R 1}+e_{(n)}
$$

where $\gamma$ is a positive real such that $\gamma \in\left[0 ; 1\left[\right.\right.$ and $e_{(n)}$ is a white circular complex Gaussian noise with variance such that $\sigma_{e}^{2}=$ $\left(1-\gamma^{2}\right) \cdot \sigma_{\alpha}^{2}$. According to (4), the AR1-coefficient $\gamma$ verifies

$$
\gamma=\frac{R_{\alpha^{A R 1}[1]}}{R_{\alpha^{A R 1}}[0]}
$$

The specific equations of the AR1-KF can be found in [17].

\subsubsection{Correlation Matching criterion [1][3]}

The CM criterion consists in imposing that the autocorrelation coefficients $R_{\alpha^{A R 1}}[\mathrm{~m}]$ of the approximated AR1 process $\alpha^{A R 1}$ perfectly match the sampled autocorrelation function $R_{\alpha}[m]$ (which is a Bessel function) of the true CG $\alpha$, for lags $^{2} m \in\{-1,0,1\}$. Using the hermitian symmetry of a stationary correlation matrix, it results that $R_{\alpha^{A R 1}}[0]=R_{\alpha}[0]$, and $R_{\alpha^{A R 1}}[1]=R_{\alpha}[1]$. Regarding equations (5)\&(3) the AR1coefficient $\gamma$ noted $\gamma_{C M}$ results in

$$
\gamma_{C M}=J_{0}\left(2 \pi f_{d} T\right)
$$

\footnotetext{
${ }^{2}$ For a more general AR-p model (with then more degrees of freedom than the AR1-model), the coefficients of the AR-p process are calculated [1] by still imposing the correlation matching constraint for lags $m \in\{-p, \ldots .,-1,0,1, \ldots, p\}$, but followed by the resolution of the YuleWalker equations in order to minimize the prediction error (model noise).
} 
It is shown in $[11,12]$ that for low Doppler and medium SNR, the asymptotic variance of the AR1(CM)-Kalman Filter is:

$$
\sigma_{\varepsilon}^{2}(A R 1(C M)) \approx \sigma_{N}^{2}+\frac{\pi}{\sqrt{2}} f_{d} T \sigma_{\alpha} \sigma_{N}
$$

This standard choice of $J_{0}\left(2 \pi f_{d} T\right)$ for $\gamma$ results from the $\mathrm{CM}$ constraint. However, note that imposing the matching of three taps (for $p=1$ ) of the approximate and original processes auto-correlation functions does not ensure the best similitude (in terms of euclidean distance for example) between the two functions. This is especially true for low $f_{d} T<<1$ (see [3], Fig. 1) where the three taps are very close to the value 1 (since $J_{0}\left(2 \pi f_{d} T\right) \approx 1-\frac{1}{4} \cdot\left(2 \pi f_{d} T\right)^{2}$ ).

\subsubsection{Minimum Asymptotic Variance criterion [11, 12]}

For low Doppler and low SNR, $[11,12]$ showed that the AR1coefficient $\gamma$ noted $\gamma_{M A V}$ that allows to minimize the asymptotic estimation error variance $\sigma_{\varepsilon}^{2}$ of the AR1-KF is

$$
\gamma_{M A V}=\sqrt{1-4\left(\left(\pi f_{d} T\right)^{4} \sigma_{N}^{2} / \sigma_{\alpha}^{2}\right)^{\frac{1}{3}}}
$$

and the closed-form expression of the corresponding asymptotic estimation error variance is

$$
\sigma_{\varepsilon}^{2}(A R 1(M A V)) \approx \frac{3}{2} \cdot\left(\sigma_{\alpha}^{2}\right)^{\frac{1}{3}} \cdot\left(\pi f_{d} T \sigma_{N}^{2}\right)^{\frac{2}{3}}
$$

\subsection{Or2-Kalman Filter}

\subsubsection{Dynamical model for approaching the $C G$ variation}

In case of slow fading, the CG variations look linear during a few symbols. It could then be more appropriate to consider 2nd-order model including a (slightly mobile) linear drift $\delta$, where the Jakes'process $\alpha_{(n)}$ is approached by $\tilde{\alpha}_{(n)}$ as

$$
\begin{aligned}
& \tilde{\alpha}_{(n)}=\tilde{\alpha}_{(n-1)}+\delta_{(n-1)} \\
& \delta_{(n)}=\delta_{(n-1)}+u_{(n)}
\end{aligned}
$$

where $u_{(n)}$ is zero mean Gaussian complex circular with a variance $\sigma_{u}^{2}$. And the parameter of this model, $\sigma_{u}^{2}$, has to be calibrated such that the slope (or drift) of the CG variation, $\delta_{(n)}$, changes slowly with time $n$, according to the actual value of $f_{d} T$. The 2 nd-order model of the CG evolution can be re-formulated in a state-space model. The state vector to be considered includes the CG and the drift, $\boldsymbol{a}_{(n)}=\left[\tilde{\alpha}_{(n)}, \boldsymbol{\delta}_{(n)}\right]^{T}$. The state evolution matrix is $\mathbf{M}=\left[\begin{array}{ll}1 & 1 \\ 0 & 1\end{array}\right]$ and the state-noise vector is $\mathbf{u}_{(n)}=\left[0, u_{(n)}\right]^{T}$. The observation matrix with size $1 \times 2$ is $\mathbf{S}_{(n)}=\mathbf{s}^{T} \stackrel{\text { def }}{=}[1,0]$. The state evolution (10)\&(11) and the observation (1) become in a state-space formulation:

$$
\begin{aligned}
& \boldsymbol{a}_{(n)}=\mathbf{M} \boldsymbol{a}_{(n-1)}+\mathbf{u}_{(n)} \\
& y_{(n)}=\mathbf{s}^{T} \boldsymbol{a}_{(n)}+N_{(n)}
\end{aligned}
$$

\subsubsection{Equations of the Or2-Kalman Filter}

Regarding the state-space formulation (12)\&(13), the two stages of the so called Or2-Kalman Filter are given by [17]:

Time Update Equations:

$$
\begin{aligned}
& \hat{\boldsymbol{a}}_{(n \mid n-1)}=\mathbf{M} \hat{\boldsymbol{a}}_{(n-1 \mid n-1)} \\
& \mathbf{P}_{(n \mid n-1)}=\mathbf{M} \mathbf{P}_{(n-1 \mid n-1)} \mathbf{M}^{H}+\mathbf{U}
\end{aligned}
$$

Measurement Update Equations:

$$
\begin{aligned}
\mathbf{K}_{(n)} & =\frac{\mathbf{P}_{(n \mid n-1)} \mathbf{s}}{\mathbf{s}^{T} \mathbf{P}_{(n \mid n-1)} \mathbf{s}+\sigma_{N}^{2}} \\
\hat{\boldsymbol{a}}_{(n \mid n)} & =\hat{\boldsymbol{a}}_{(n \mid n-1)}+\mathbf{K}_{(n)}\left(y_{(n)}-\mathbf{s}^{T} \hat{\boldsymbol{a}}_{(n \mid n-1)}\right) \\
\mathbf{P}_{(n \mid n)} & =\left(\mathbf{I}_{2}-\mathbf{K}_{(n)} \mathbf{s}^{T}\right) \mathbf{P}_{(n \mid n-1)}
\end{aligned}
$$

where $\mathbf{K}_{(n)}=\left[\begin{array}{l}k_{1(n)} \\ k_{2(n)}\end{array}\right]$ is the Kalman gain vector, $\mathbf{U}=\left[\begin{array}{cc}0 & 0 \\ 0 & \sigma_{u}^{2}\end{array}\right]$, and $\mathbf{P}_{(n \mid n)}$ and $\mathbf{P}_{(n \mid n-1)}$ are respectively the $2 \times 2$ a posteriori and predicted error covariance matrices.

\section{ANALYSIS AND OPTIMIZATION OF THE OR2-KF}

\subsection{Steady-state Or2-KF equations}

Since the linear system (12)\&(13) is observable and controllable, an asymptotic regime is quickly reached ([17]), for which the Kalman gain and covariance matrices become constant. For our second-order model, we can write for sufficiently large $n$ that $\mathbf{K}_{(n)}=\mathbf{K}_{(n+1)}=\mathbf{K}_{\infty} \stackrel{\text { def }}{=}\left[\begin{array}{l}k_{1} \\ k_{2}\end{array}\right]$, and $\mathbf{P}_{(n \mid n)}=\mathbf{P}_{(n+1 \mid n+1)}=\mathbf{P}_{\infty} \stackrel{\text { def }}{=}\left[\begin{array}{ll}P_{11} & P_{12} \\ P_{21} & P_{22}\end{array}\right]$, and again $\mathbf{P}_{(n \mid n-1)}=\mathbf{P}_{(n+1 \mid n)}=\mathbf{P}_{\infty} \stackrel{\text { def }}{=}\left[\begin{array}{ll}P_{11}^{\prime} & P_{12}^{\prime} \\ P_{21}^{\prime} & P_{22}^{\prime}\end{array}\right]$. Using equations (14)\&(17), we check that the KF in asymptotic regime (or tracking mode) is reduced to a time-invariant linear recursive filter defined by:

$$
\begin{aligned}
\hat{\alpha}_{(n \mid n)} & =\hat{\alpha}_{(n-1 \mid n-1)}+\hat{\delta}_{(n-1 \mid n-1)}+k_{1} \cdot v_{\varepsilon(n)} \\
\hat{\delta}_{(n \mid n)} & =\hat{\delta}_{(n-1 \mid n-1)}+k_{2} \cdot v_{\varepsilon(n)} \\
\text { with } v_{\varepsilon(n)} & \stackrel{\text { def }}{=} y_{(n)}-\left(\hat{\alpha}_{(n-1 \mid n-1)}+\hat{\delta}_{(n-1 \mid n-1)}\right)
\end{aligned}
$$

The filter is equivalent to a second-order tracking loop parametrized by 2 constants $k_{1}$ and $k_{2}$, that play respectively the role of the Proportional (P) and Integral (I) gains of the loop ([13][14][10]). But here the values of these 2 parameters can not directly be chosen: they depend on the channel state $\left(f_{d} T, S N R=\sigma_{\alpha}^{2} / \sigma_{N}^{2}\right)$ and on the model noise variance $\sigma_{u}^{2}$, which is the only parameter that the user can tune. In the following, we aim to express $\left(k_{1}, k_{2}\right)$ and then the asymptotic error variance $\sigma_{\varepsilon}^{2}$ with respect to $\sigma_{u}^{2}$, in the perspective of an optimization. 


\subsection{Steady-state Kalman gain}

To find the steady-state Kalman gain vector $\left(k_{1}, k_{2}\right)$ we need first to find the matrix $\mathbf{P}_{\infty}$, and then to resolve the so-called Riccati equations (16)\&(15)\&(18) rewritten for $n \rightarrow \infty$ as:

$$
\begin{aligned}
k_{1} & =\frac{P_{11}^{\prime}}{P_{11}^{\prime}+\sigma_{N}^{2}} \quad \text { and } k_{2}=\frac{P_{21}^{\prime}}{P_{11}^{\prime}+\sigma_{N}^{2}} \\
{\left[\begin{array}{ll}
P_{11}^{\prime} & P_{12}^{\prime} \\
P_{21}^{\prime} & P_{22}^{\prime}
\end{array}\right] } & =\left[\begin{array}{cc}
P_{11}+P_{12}+P_{21}+P_{22} & P_{12}+P_{22} \\
P_{21}+P_{22} & P_{22}+\sigma_{u}^{2}
\end{array}\right] \\
{\left[\begin{array}{ll}
P_{11} & P_{12} \\
P_{21} & P_{22}
\end{array}\right] } & =\left[\begin{array}{cc}
\left(1-k_{1}\right) P_{11}^{\prime} & (1-k 1) P_{12}^{\prime} \\
-k_{2} P_{11}^{\prime}+P_{21}^{\prime} & -k_{2} P_{12}^{\prime}+P_{22}^{\prime}
\end{array}\right]
\end{aligned}
$$

We can easily deduce that $k_{2} P_{11}^{\prime}=k_{1} P_{21}^{\prime}, k_{1} P_{12}^{\prime}=P_{22}$ and $k_{2} P_{12}^{\prime}=\sigma_{u}^{2}$ and thus (using the hermitian symmetry of the covariance matrices and (22)) that $k_{1}, k_{2}, \mathbf{P}_{\infty}$, and $\mathbf{P}_{\infty}$ are real-valued objects. It results that $P_{21}^{\prime}=P_{12}^{\prime}=\sigma_{u} \sqrt{P_{11}^{\prime}+\sigma_{N}^{2}}$, and equation (22) provides an exact expression of the KF gain with respect to $P_{11}^{\prime}$ and $\sigma_{N}^{2}$, where $P_{11}^{\prime}$ can be found after some manipulations of the Riccati equations as the solution of ([14]):

$$
P_{11}^{\prime 4}=\sigma_{u}^{2}\left(P_{11}^{\prime}+\sigma_{N}^{2}\right)\left(P_{11}^{\prime}+2 \sigma_{N}^{2}\right)^{2}
$$

Now, in order to obtain more tractable formulation, we make the assumption $P_{11}^{\prime}<<\sigma_{N}^{2}$, which means that we have a low Kalman gain $k_{1}<<1$ (see (22)). This assumption a priori agrees with a low Doppler $\left(f_{d} T \leq 10^{-2}\right)$ and low $\operatorname{SNR}\left(\sigma_{N} \in\right.$ $[0.01,1])$ scenario. We get then from (25) the approximation $P_{11}^{\prime} \approx \sigma_{N}^{2} \sqrt{2 \sigma_{u} / \sigma_{N}}$, which is injected in (22) to finally obtain:

$$
\begin{aligned}
& k_{1} \approx \frac{P_{11}^{\prime}}{\sigma_{N}^{2}} \approx \sqrt{\frac{2 \sigma_{u}}{\sigma_{N}}} \\
& k_{2} \approx \frac{P_{21}^{\prime}}{\sigma_{N}^{2}} \approx \frac{\sigma_{u}}{\sigma_{N}}
\end{aligned}
$$

Note that $k_{2}, k_{1}$ are then linked by $k_{1} \approx \sqrt{2 k_{2}}$. Note also that our assumption implies that the model noise is chosen weak compared to the observation noise, i.e. $\sigma_{u}^{2}<<\sigma_{N}^{2}$ (see (26)).

\subsection{Steady-state MSE and optimization}

\subsubsection{Steady-state transfer function of the KF and MSE}

Using (20)\&(21), the equation (19) of the steady-state Kalman Filter can be expressed in Z-Transform domain by:

$$
\hat{\alpha}(z) \cdot\left[1-z^{-1}\right]=\left[k_{1}+\frac{k_{2} \cdot z^{-1}}{1-z^{-1}}\right] \cdot v_{\varepsilon}(z)
$$

The error signal (21) can be rewritten versus the estimation error from $(21) \&(19) \&(1)$ as

$$
v_{\varepsilon(n)}=\frac{1}{1-k_{1}} \cdot\left\{\alpha_{(n)}-\hat{\alpha}_{(n \mid n)}\right\}+\frac{1}{1-k_{1}} \cdot N_{(n)}
$$

Combining (29) and (28) leads in the Z-transform domain to $\hat{\alpha}(z)=L(z) \cdot(\alpha(z)+N(z))$ and then to the estimation error

$$
\varepsilon(z)=\alpha(z)-\hat{\alpha}(z)=(1-L(z)) \cdot \alpha(z)-L(z) \cdot N(z)
$$

where $L(z)$ is the transfer function of the steady-state KF:

$$
L(z)=\frac{\frac{k_{1}-k_{2}}{1-k_{1}} \cdot\left(1-z^{-1}\right)+\frac{k_{2}}{1-k_{1}}}{\left(1-z^{-1}\right)^{2}+\frac{k_{1}-k_{2}}{1-k_{1}} \cdot\left(1-z^{-1}\right)+\frac{k_{2}}{1-k_{1}}}
$$

In using the previous assumptions, we have that $0<$ $k_{2}<<k_{1} \approx \sqrt{2 k_{2}}<<1$, and then $L(z)$ is a low-pass filter which can be approximated in frequency domain (using $z=e^{j 2 \pi f T}$ and then $1-z^{-1} \approx j 2 \pi f T$ for $f T<<1$ ) by

$$
L\left(e^{j 2 \pi f T}\right) \approx \frac{\sqrt{2 k_{2}} \cdot(j 2 \pi f T)+k_{2}}{(j 2 \pi f T)^{2}+\sqrt{2 k_{2}} \cdot(j 2 \pi f T)+k_{2}}
$$

This corresponds to a second-order low-pass filter with normalized natural pulsation $\omega_{n} T \approx \sqrt{k_{2}} \approx \frac{k_{1}}{\sqrt{2}}$, and with imposed damping factor $\zeta=\frac{\sqrt{2}}{2}$.

Using (30), the asymptotic MSE is divided into two parts:

$$
\sigma_{\varepsilon}^{2}=\sigma_{\varepsilon \alpha}^{2}+\sigma_{\varepsilon N}^{2}
$$

- the dynamic error variance $\sigma_{\varepsilon \alpha}^{2}$ is due to the variations of the CG $\alpha_{(n)}$ filtered by the high pass filter $1-L(z)$ :

$$
\begin{array}{r}
\sigma_{\varepsilon \alpha}^{2} \stackrel{\text { def }}{=} \int_{-\frac{1}{2 T}}^{+\frac{1}{2 T}}\left|1-L\left(e^{j 2 \pi f T}\right)\right|^{2} \cdot \Gamma_{\alpha}(f) d f \\
\approx \int_{-f_{d}}^{+f_{d}} \frac{(2 \pi f T)^{4}}{k_{2}^{2}} \cdot \Gamma_{\alpha}(f) d f=\frac{3}{8} \cdot \sigma_{\alpha}^{2} \cdot \frac{\left(2 \pi f_{d} T\right)^{4}}{k_{2}^{2}}
\end{array}
$$

The approximation in (34) is made in using the previous approximation of $L($.$) in (32), and in assuming low$ Doppler $f_{d} T<<1$, and then a Kalman gain (or a natural pulsation) such that $2 \pi f_{d} T<<\sqrt{k_{2}}<<1$. The exact integration is then computed from $\Gamma_{\alpha}(f)$ defined in (2) by the way of the variable change $\cos \theta=\left(f / f_{d}\right)$.

- the static error variance $\sigma_{\varepsilon N}^{2}$ is due to the additive noise $N_{(n)}$ filtered by the low pass filter $-L(z)$ :

$$
\begin{aligned}
\sigma_{\varepsilon N}^{2} & \stackrel{\text { def }}{=} \sigma_{N}^{2} \times T \int_{-\frac{1}{2 T}}^{+\frac{1}{2 T}}\left|L\left(e^{j 2 \pi f T}\right)\right|^{2} d f \\
& =\sigma_{N}^{2} \times \frac{2 k_{1}^{2}-3 k_{1} k_{2}+2 k_{2}}{k_{1} \cdot\left[4-\left(2 k_{1}+k_{2}\right)\right]} \\
& \approx \sigma_{N}^{2} \times \frac{3 \sqrt{2}}{4} \cdot \sqrt{k_{2}}
\end{aligned}
$$

The exact integration (36) is obtained from exact $L(z)$ in (31) in using method of the book [16]. The approximation in (37) is obtained in using $2 k_{2} \approx k_{1}^{2}$ and $k_{1}^{2}<<k_{1}<<1$. 


\subsubsection{Optimal Or2-parameter under MAV criterion}

Inserting the approximation (27) of $k_{2} \approx \sigma_{u} / \sigma_{N}$ in (34)\&(37), we see that the dynamic component $\sigma_{\varepsilon \alpha}^{2}$ is inversely proportional to $\sigma_{u}^{2}$, whereas the static component $\sigma_{\varepsilon N}^{2}$ is directly proportional to $\sigma_{u}^{\frac{1}{2}}$. Now the model noise variance $\sigma_{u}^{2}$ that permits (for $2 \pi f_{d} T<\sqrt{\frac{\sigma_{u}}{\sigma_{N}}}<<1$ ) minimizing the global MSE $\sigma_{\varepsilon}^{2}$ in (33) in assuming (34)\&(37), can be calculated as

$$
\sigma_{u}^{2}(O r 2(M A V))=\left(4 \cdot\left(2 \pi f_{d} T\right)^{16} \cdot\left(\sigma_{\alpha}^{2}\right)^{4} \cdot \sigma_{N}^{2}\right)^{\frac{1}{5}}
$$

And the corresponding optimal MSE results in

$$
\sigma_{\varepsilon}^{2}(O r 2(M A V))=\frac{15}{8}(\sqrt{2} \pi)^{\frac{4}{5}} \cdot\left(\sigma_{\alpha}^{2}\right)^{\frac{1}{5}} \cdot\left(\sigma_{N}^{2} \cdot f_{d} T\right)^{4 / 5}
$$

\section{SIMULATION RESULTS AND DISCUSSION}

Fig. 2 and Fig. 3 compare the MSE obtained with the AR1$\mathrm{KF}$ (with CM and MAV criteria) and with the Or2-KF (with MAV criterion) by the way of Monte-Carlo simulations. We also plot the on-line BCRB ([8]) as reference. We verify by these figures that:

- With the CM criterion, the MSE of the AR1-KF is approximately constant with respect to the Doppler frequency for $f_{d} T \leq 10^{-2}$ (which agrees with (7) for medium SNR), and is far from the BCRB for lower $f_{d} T$, as was observed in [9][10]. Furthermore, the use of the MAV versus CM criterion improves the AR1-KF strongly, especially for lower Doppler frequencies and low SNRs, which corroborates the study of [11, 12].

- For the Or2-KF, the MSE computed by Monte-Carlo simulation is very close to $\sigma_{\varepsilon}^{2}(\operatorname{Or} 2(M A V))$ obtained by the closed-form expression (39), so we validate our theoretical analysis and our approximations.

- The most important point is that with the proposed Or2$\mathrm{KF}$ and its optimization, we are closer to the BCRB than with the AR1-KF (under CM or MAV criteria), especially for lower normalized Doppler frequencies $f_{d} T \leq 10^{-2}$. This point reveals the advantage of using an appropriate 2nd-order modeling versus a first-order one in slow fading scenario, as we had already pointed out in [10]. The present study shows that this 2nd-order model allows a KF MSE that is proportional to the $\left(\frac{4}{5}\right)$ power of $f_{d} T$, versus the $\left(\frac{2}{3}\right)$ power for the 1 st-order AR model, in agreement with (39) and (9).

\section{CONCLUSION}

The modeling for the Kalman Filter based Rayleigh channel estimation was investigated, together with the tuning of the (a) $f_{d} T=10^{-4}$

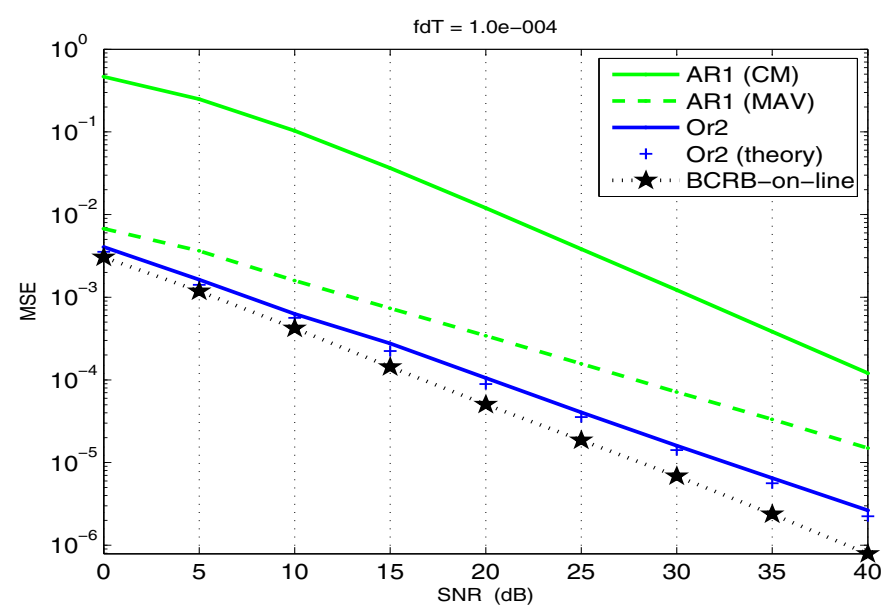

(b) $f_{d} T=10^{-3}$

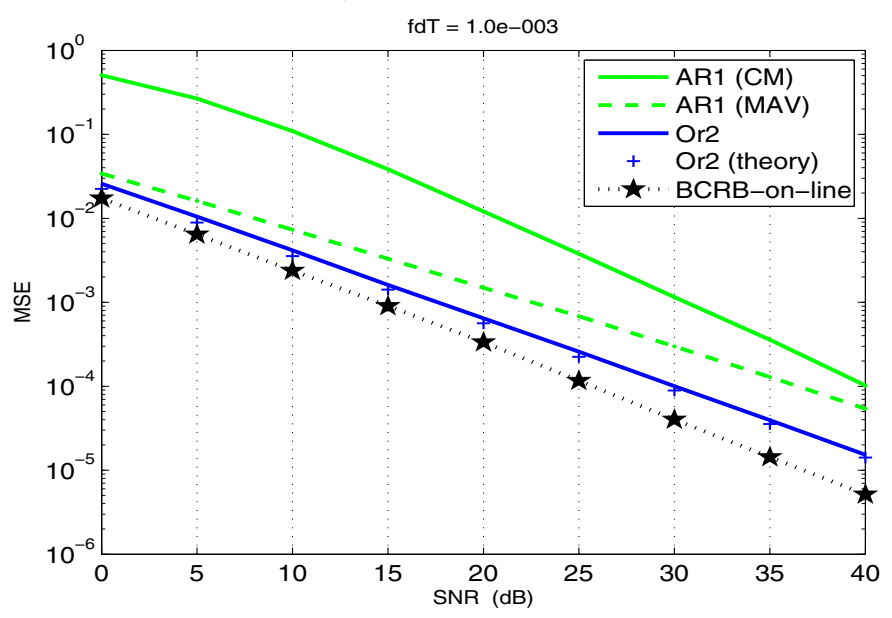

(c) $f_{d} T=10^{-2}$

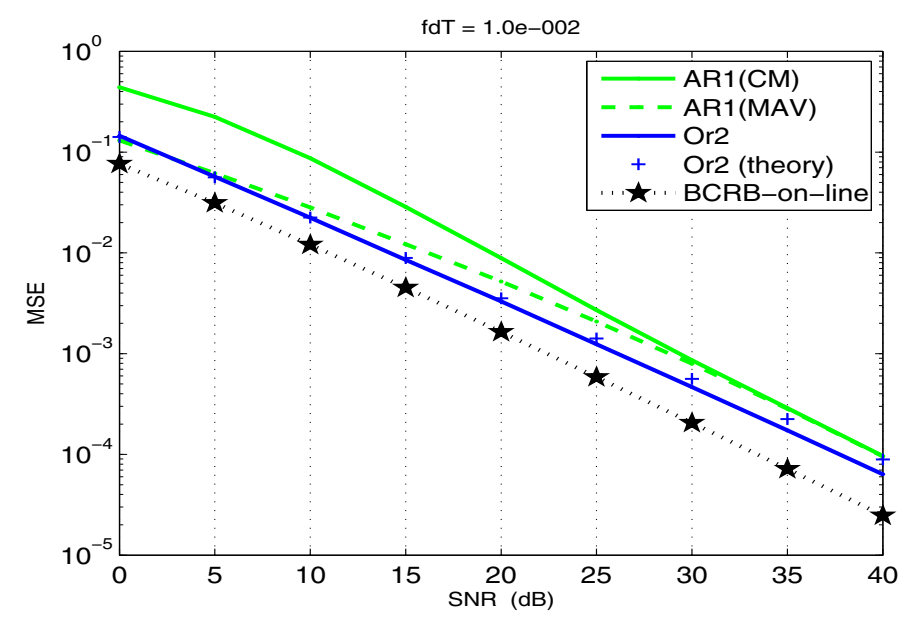

Fig. 2. MSE of the Kalman Filters for the proposed Or2(MAV)-KF compared to literature AR1(CM)-KF and AR1(MAV)-KF versus SNR for $f_{d} T=10^{-4}$ (a), $f_{d} T=10^{-3}$ (b), and $f_{d} T=10^{-2}$ (c) 


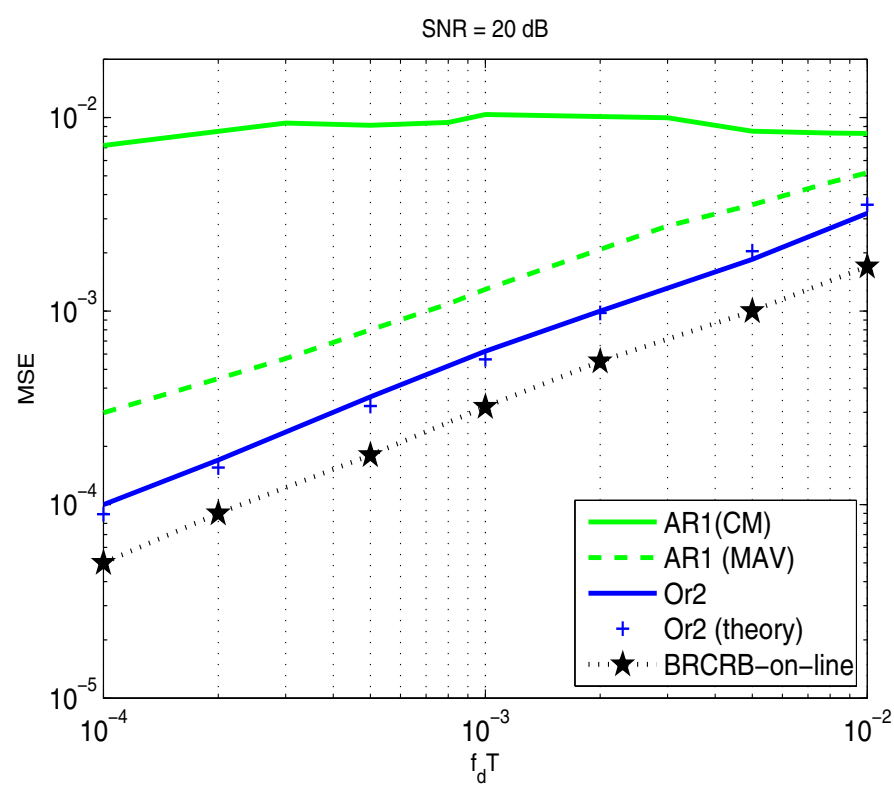

Fig. 3. MSE in function of $f_{d} T$, for $S N R=20 \mathrm{~dB}$.

parameters $\left(\gamma\right.$ or $\sigma_{u}^{2}$ ) of the models. The simple case of a flat fading channel was considered, but the results can be applied or generalized to more complex systems. In the literature, the 1st-order Auto-Regressive (AR1) model is often used, with a Bessel AR1-coefficient resulting from the standard Correlation Matching criterion. This choice seems to be convenient for high Doppler frequencies and high SNR, but not for lower ones. For most conventional Doppler speeds $\left(f_{d} T \leq 10^{-2}\right)$, we have demonstrated the interest of using a specific 2ndorder model (based on a Brownian evolution of the Complex Gain slope) versus 1st-order model. We have given closed form expressions of the optimal model-parameter that minimizes the asymptotic estimation error variance assuming Rayleigh-Jakes channel. It is demonstrated that the MSE of the 2nd-order KF changes proportionally to the $\left(\frac{4}{5}\right)$ power of the product $\left(f_{d} T \times \sigma_{N}^{2}\right)$, where $\sigma_{N}^{2}$ is the observation noise variance. Simulation results proved an amelioration in terms of MSE, in the choice we proposed.

\section{REFERENCES}

[1] K. E. Baddour and N. C. Beaulieu, "Autoregressive modeling for fading channel simulation", in IEEE Trans. Wireless Comm., vol. 4, no. 4, pp. 1650-1662, July 2005.

[2] H.S. Wang and P.C. Chang, "On verifying the First Order Markovian Assumption for a Rayleigh Fading Channel Model", in IEEE Trans. on Vehicular Tech., vol. 45, no. 2, pp. 353-357, May 1996.

[3] C. Komninakis, C. Fragouli, A.H. Sayed, and R.D. Wesel, "Multi-Input Multi-Output Fading Channel Tracking and Equalization Using Kalman Estimation", in IEEE Trans. Signal Proc., vol. 50, no. 5, pp. 1065-1076, May 2002.
[4] Z. Liu, X. Ma, and G.B. Giannakis, "Space-time coding and Kalman Filtering for time selective fading channels", in IEEE Trans. on Comm., vol. 50, no. 2, February 2002.

[5] W. Chen and R. Zhang, "Kalman filter Channel estimator for OFDM systems in time and frequency-selective fading environment" in Proc. IEEE ICASSP, vol. 4, pp. 17-21, Montreal, Canada, May 2004.

[6] T.Y. Al-Naffouri, "An EM-based Forward-Backward Kalman Filter for the Estimation of Time-Variant Channels in OFDM" in IEEE Trans. Signal Proc., vol. 55, no. 7, pp. 3924-3930, July 2007.

[7] H. Hijazi and L. Ros, "Joint Data QR-Detection and Kalman Estimation for OFDM Time-varying Rayleigh Channel Complex Gains" in IEEE Trans. Comm., vol. 58, no. 1, pp. 170-178, January 2010.

[8] H. Hijazi and L. Ros, "Bayesian Cramer-Rao Bounds for OFDM Slowly Varying Multi-path Rayleigh Channel Estimation" in ELSEVIER Signal Proc. Fast Communication, vol. 89, pp. 111-115, January 2009.

[9] H. Abeida, J.M. Brossier, L. Ros and J. Vilà-Valls, "An EM algorithm for path delay and complex gain estimation of a slowly varying fading channel for CPM signals", in Proc. IEEE Globecom, pp. 47-52, Hawaï, Dec. 2009.

[10] L. Ros, H. Hijazi and E.P. Simon "Paths Complex Gain Tracking Algorithms for OFDM Receiver in SlowlyVarying Channels" in Proc. IEEE ISCCSP, pp. 1-6, Limassol, Cyprus, March 2010.

[11] S. Ghandour, "Estimation de canal vanouissement lent pour les communications radio-mobiles", Master's Research Report. June 2010.

[12] S. Ghandour-Haidar, L. Ros, J.M. Brossier, "On the Use of first-order Autoregressive Modeling for Rayleigh Flat Fading Channel Estimation with Kalman filter", submitted in Elsevier Signal Processing.

[13] A. Patapoutian, "On phase-locked loops and Kalman filters" in IEEE Trans. Comm., vol. 47, pp. 670-672, May 1999.

[14] Y. Qian, X. Cui, M. Lu, Z. Feng, "Steady-State Performance of Kalman Filter for DPLL" in Tsinghua Science and Technology, vol. 14, no. 4, pp. 470-473, August 2009.

[15] W. C. Jakes, Microwave Mobile Communications. IEEE Press, 1983.

[16] E.I. Jury, Theory and Application of the Z-Transform Method. Wiley, New York, 1964.

[17] S.M. Kay, Fundamentals of Statistical Signal Processing: Estimation Theory. Prentice Hall PTR, 1993. 